\title{
Protein phosphatase 4 promotes hepatic lipogenesis through dephosphorylating acetyl-CoA carboxylase 1 on serine 79
}

\author{
XIANGYU MENG ${ }^{1,2}$, MENG LI ${ }^{1,2}$, JUN GUO $^{1,2}$, WEIQING TANG ${ }^{2}$, SHU WANG $^{2}$, \\ YONG MAN $^{2}$, XIUQING HUANG ${ }^{2}$ and JIAN LI ${ }^{1,2}$ \\ ${ }^{1}$ Peking University Fifth School of Clinical Medicine, Beijing Hospital; ${ }^{2}$ The Key Laboratory of Geriatrics, \\ Beijing Hospital and Beijing Institute of Geriatrics, Ministry of Health, Beijing 100730, P.R. China
}

Received November 5, 2013; Accepted May 9, 2014

DOI: $10.3892 / \mathrm{mmr} .2014 .2397$

\begin{abstract}
Reversible phosphorylation has a critical role in the regulation of the activity of acetyl-CoA carboxylase 1 (ACC1), which is associated with de novo lipogenesis. It has been shown that AMP-activated protein kinase (AMPK) phosphorylates ACC1 on serine 79 and inhibits its activity; however, the mechanism of $\mathrm{ACCl}$ dephosphorylation remains elusive. Protein phosphatase 4 (PP4), a ubiquitous serine/threonine phosphatase, regulates a variety of cellular functions; however, whether PP4 is involved in lipid metabolism has yet to be elucidated. In the present study, PP4 was identified as a novel regulator of $\mathrm{ACC} 1$, which is also involved in hepatic lipogenesis. The expression of PP4 was found to be significantly increased in the livers of $\mathrm{db} / \mathrm{db}$ mice. Furthermore, pACC1-Ser79/ACC1 levels were observed to be decreased and high triglyceride accumulation was found in the livers of $\mathrm{db} / \mathrm{db}$ mice. Moreover, PP4 overexpression was observed to lead to a decreased pACC1-Ser79/ACC1 ratio and subsequently an increased intracellular triglyceride content in mouse primary hepatocytes. PP4 was also found to directly interact with pACC1-Ser79 in human HepG2 cells. In conclusion, the present study showed that PP4 may be a novel regulator in hepatic lipogenesis through dephosphorylating ACC1 on serine 79, suggesting that PP4 may be a promising therapeutic target in lipid metabolism disorders.
\end{abstract}

\section{Introduction}

Epidemiological investigation has shown that nonalcoholic fatty liver disease (NAFLD) is an important public health

Correspondence to: Dr Jian Li or Mrs Xiuqing Huang, The Key Laboratory of Geriatrics, Beijing Hospital and Beijing Institute of Geriatrics, Ministry of Health, 1 Dahua Road, Beijing 100730, P.R. China

E-mail: 1ijian@bjhmoh.cn

E-mail: huang_xq118@126.com

Key words: protein phosphatase 4, acetyl-CoA carboxylase 1, triglyceride accumulation problem. NAFLD develops and progresses to more advanced forms, including steatohepatitis, fibrosis and cirrhosis $(1,2)$. Surplus lipid, particularly triglyceride deposition, is a prerequisite for the development of NAFLD. Furthermore, a persistent increase in triglyceride synthesis is an important factor in the accumulation of fatty acids in the liver.

Mammalian acetyl-CoA carboxylase 1 (ACC1) catalyzes the carboxylation of acetyl-CoA to form malonyl-CoA, an intermediate in the de novo synthesis of fatty acids (3). Chang et al (4) found that the activity of ACC1 was markedly increased in obese mice. It has been proposed that the phosphorylation and/or dephosphorylation of target serine residues is important for the short-term regulation of ACC1 (5-7). Moreover, AMP-activated protein kinase (AMPK) phosphorylates ACC1 on serine 79, which further inhibits its activity, while dephosphorylation of ACC1 on serine 79 increases its activity $(8,9)$. Various studies have reported that protein phosphatases may dephosphorylate ACC1 (8-10); however, only a few have shown that ACC1 may be dephosphorylated by protein phosphatase-2A (PP2A) (8). It is not clear whether other protein phosphatases are involved in ACC1 dephosphorylation.

The serine/threonine phosphatase phosphatase 4 (PP4) is an important member of the PP2A family. PP4 is highly conserved from invertebrates to vertebrates (11). PP4 dephosphorylates a series of downstream substrates through its phosphatase activity and participates in various signaling pathways, including the nuclear factor $\kappa$-light-chain-enhancer of activated B cells (NF- $\kappa \mathrm{B})$ pathway, apoptosis, hepatic gluconeogenesis and histone modification (12-15). However, whether PP4 is involved in lipid metabolism has yet to be elucidated.

Therefore, the present study investigated the expression of PP4, ACC1 and pACC1-Ser79 in the livers of $\mathrm{db} / \mathrm{db}$ mice and mouse primary hepatocytes.

\section{Materials and methods}

Animals. Six to eight week-old male $\mathrm{db} / \mathrm{db}$ mice were purchased from the National Resource Center for Mutant Mice (Nanjing, China) which were originally purchased from The Jackson Laboratory (Bar Harbor, ME, USA). C57BL/6 mice were purchased from Vital River Laboratory Animal 
Technology Co., Ltd. China (Beijing, China). Animals were fed a standard laboratory diet for two weeks in a temperature$\left(20-24^{\circ} \mathrm{C}\right)$ and humidity-controlled (45-55\%) environment under a 12-h light/dark cycle. All animal protocols were approved by the Animal Ethics Committee at the Beijing Institute of Geriatrics (Beijing, China).

Adenovirus vector construction. The pAdxsi-GFP-PP4 adenovirus vector (PP4-Ad) and the corresponding control adenovirus vector (Control-Ad) were purchased from the Chinese National Human Genome Center (Beijing, China).

Cell culture. HepG2 cells (American Type Culture Collection, Rockville, MD, USA) were cultured in low-glucose Eagle's minimum essential medium (Invitrogen Life Technologies, St. Louis, MO, USA) supplemented with $10 \%$ fetal bovine serum (Hyclone, Waltham, MA, USA), $100 \mathrm{U} / \mathrm{ml}$ penicillin (Invitrogen Life Technologies) and $0.1 \mathrm{mg} / \mathrm{ml}$ streptomycin (Invitrogen Life Technologies). Cells were maintained at $37^{\circ} \mathrm{C}$ in humidified air with $5 \% \mathrm{CO}_{2}$.

Primary hepatocyte isolation. The isolation of primary hepatocytes was performed as described previously $(16,17)$. In brief, the mice were anesthetized using intraperitoneal injection with $4 \%$ chloral hydrate (100 $\mu \mathrm{l} / 100 \mathrm{~g}$ body weight) and perfused through the vena porta hepatis with D-Hanks solution, then with collagenase solution at $37^{\circ} \mathrm{C}$ for $10 \mathrm{~min}$. Livers were carefully removed and transferred onto a Petri dish, then cut into small pieces and pressed through a sieve and flushed with warm Dubecco's modified Eagle's medium/F12. Centrifugation was performed at $24 \mathrm{xg}$ for $5 \mathrm{~min}$ at $4^{\circ} \mathrm{C}$. The supernatant was discarded and the hepatocyte cell pellet was retrieved.

Western blot analysis. Cell lysates (10-30 $\mu \mathrm{g}$ protein) were separated using $10 \%$ SDS-PAGE and transferred to polyvinylidene fluoride membranes (Millipore Corporation, Billerica, MA, USA), blocked using 5\% non-fat dry milk for $2 \mathrm{~h}$ and probed with primary antibodies at $4^{\circ} \mathrm{C}$ overnight. The blots were incubated with horseradish peroxidase-conjugated anti-immunoglobuin G (Millipore Corporation), followed by detection using a Fusion SL 3500 enhanced chemiluminescence system (Vilber Lourmat, Marne-la-Vallée, France). Rabbit monoclonal anti-ACC1 and anti-pACC1 (Abcam, Cambridge, MA, USA) and goat polyclonal anti-PP4 (Santa Cruz Biotechnology, Dallas, TX, USA) antibodies were purchased. Antibodies against $\beta$-actin were purchased from Cell Signaling Technology, Inc. (Beverly, MA, USA).

Co-immunoprecipitation. Cells were harvested and lysed $24 \mathrm{~h}$ after transfection [lysate buffer: $50 \mathrm{mM}$ Tris- $\mathrm{HCl}(\mathrm{pH} 8.0)$, $1 \mathrm{mM}$ EDTA, $6 \mathrm{mM}$ ethylene glycol tetraacetic acid, $1 \%$ NP-40, $120 \mathrm{mM} \mathrm{NaCl}, 1 \mathrm{mM}$ dithiothreitol, $50 \mu \mathrm{M}$ phenylmethylsulfonyl fluoride and $2 \mu \mathrm{g} / \mathrm{ml}$ aprotinin]. Endogenous PP4 was immunoprecipitated using the anti-PP4 antibody and the endogenous pACC1-Ser79 was immunoprecipitated using an anti-pACC1-Ser79 antibody at $4^{\circ} \mathrm{C}$ overnight. The immunoprecipitates were washed three times with buffer containing $50 \mathrm{mM}$ 4-(2-hydroxyethyl)-1-piperazineethanesulfonic acid (pH 7.4), $0.1 \%$ Triton X-100 and $500 \mathrm{mM} \mathrm{NaCl}$. The immuno- precipitates were then separated and analyzed using western blot analysis.

Triglyceride analysis. Intracellular triglyceride concentration was measured as described previously (18-20). Cells were washed three times with ice-cold phosphate-buffered saline and added to an isovolumic mixture of chloroform and methanol $(2: 1 \mathrm{v} / \mathrm{v})$. Subsequently, $0.05 \% \mathrm{H}_{2} \mathrm{SO}_{4}$ was added and the samples were vortexed and centrifuged to split the phases. The bottom phase was removed to $1 \%$ Triton X-100 (1:1 v/v), dried and dissolved in deionized water and analyzed using a Triglyceride Reagent kit (ShenSuoYouFu Medical Diagnostic Products Co., Ltd., Shanghai, China).

Statistics. GraphPad Prism 5 (GraphPad Software, Inc., La Jolla, CA, USA) was used for statistical analysis. All values are presented as the mean \pm standard deviation of the indicated number of measurements. Differences were analyzed using the Student's t-test. $\mathrm{P}<0.05$ was considered to indicate a statistically significant difference.

\section{Results}

PP4 is highly expressed in the livers of $d b / d b$ mice. The $\mathrm{db} / \mathrm{db}$ mice exhibited increased levels of glucose, total triglyceride and low density lipoprotein-cholesterol in the serum compared with the control groups (Fig. 1A). Severe lipid accumulation was observed in the livers of the $\mathrm{db} / \mathrm{db}$ mice as demonstrated through the elevated triglyceride levels in the livers (Fig. 1B). The expression of PP4, as well as molecules of lipid metabolism, including fatty acid synthase (FAS), sterol regulatory element-binding protein (SREBP) 1 and low density lipoprotein receptor (LDLR) were analyzed in the livers of $\mathrm{db} / \mathrm{db}$ mice and C57BL/6 mice. As shown in Fig. 1C, the levels of PP4, FAS, SREBP1 and LDLR were found to be significantly increased in the livers of the $\mathrm{db} / \mathrm{db}$ mice. Thus, PP4 may be involved in the regulation of triglyceride metabolism in the liver.

PP4 overexpression induces the accumulation of triglycerides in mouse primary hepatocytes. To determine whether PP4 overexpression induces triglyceride accumulation in mouse primary hepatocytes, adenovirus-mediated PP4 overexpression was used. As shown in Fig. 2A and B, PP4 was highly overexpressed in the mouse primary hepatocytes following treatment with PP4-Ad compared with treatment with control-Ad. In addition to an increase in PP4 expression, an increase in triglyceride content was observed (Fig. 2C), indicating that PP4 may have an important role in triglyceride accumulation.

PP4 regulates hepatic triglyceride content through regulating the dephosphorylation of ACC1. Considering the critical importance of lipogenic genes in hepatic lipid homeostasis, the levels of FAS, SREBP1, ACC1 and pACC1-Ser79 were analyzed following PP4 overexpression. As shown in Fig. 3A, PP4 overexpression in the primary hepatocytes led to a decrease in the levels of pACC1-Ser79/ACC1, while no significant change occurred in the levels of SREBP1 (Fig. 2D). Furthermore, reduced levels of pACC1-Ser79/ ACC1 were 
A

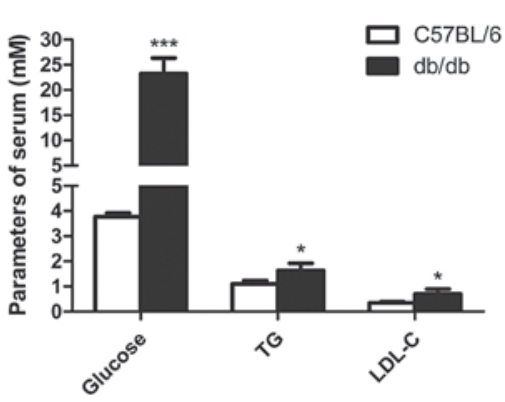

B

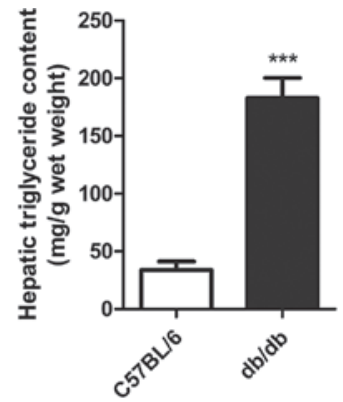

C

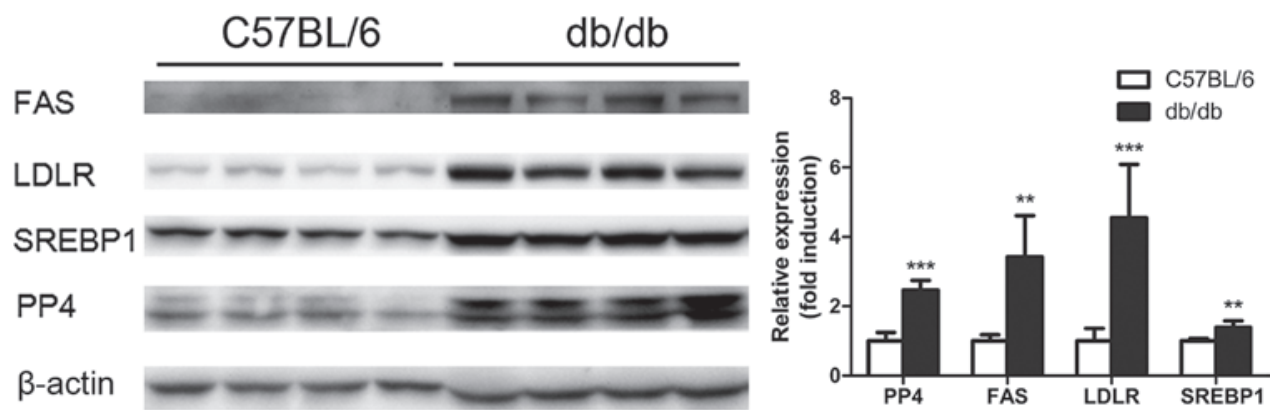

Figure 1. PP4 is highly expressed in the livers of db/db mice. (A) Compared with the C57BL/6 mice, the db/db mice showed increased levels of glucose, TG and LDL-C in the serum. (B) Intracellular triglyceride measurement revealed severe lipid accumulation in the livers of the db/db mice. (C) FAS, SREBP1 and LDLR were increased in the livers of the $\mathrm{db} / \mathrm{db}$ mice. Data are expressed as the mean \pm standard deviation. ${ }^{* * *} \mathrm{P}<0.01 ;{ }^{* * * *} \mathrm{P}<0.001 \mathrm{vs}$. control ( $\mathrm{n}=4$ ). TG, total triglyceride; LDL-C, low density lipoprotein-cholesterol; FAS, fatty acid synthase; SREBP1, sterol regulatory element-binding protein; LDLR, low density lipoprotein receptor; PP4, protein phosphatase 4.

A

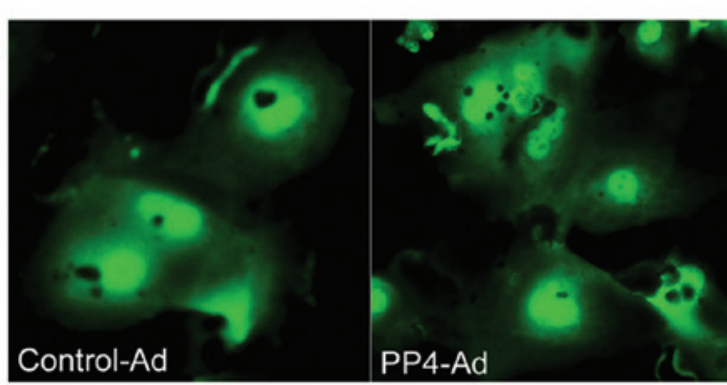

B
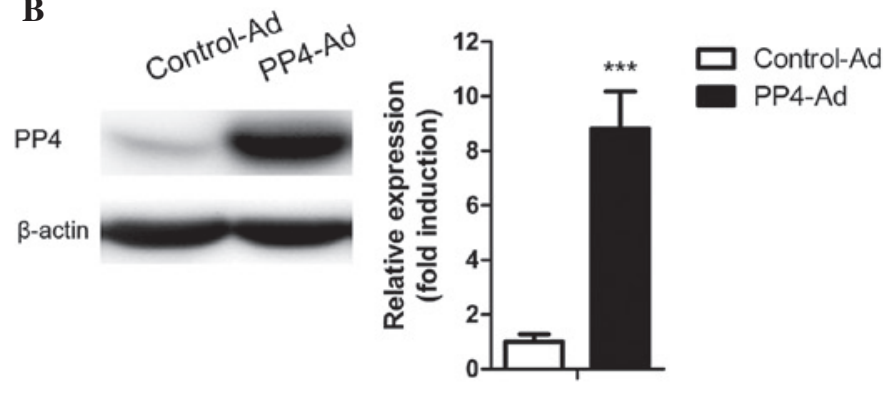

C

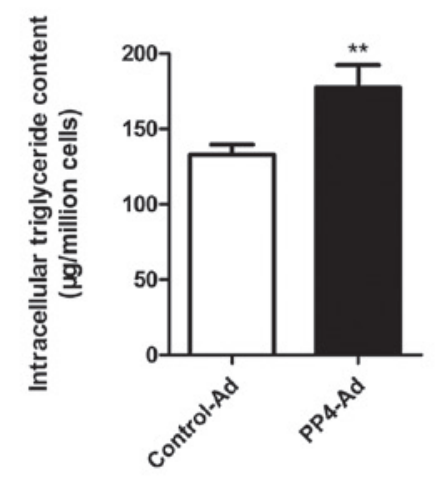

D
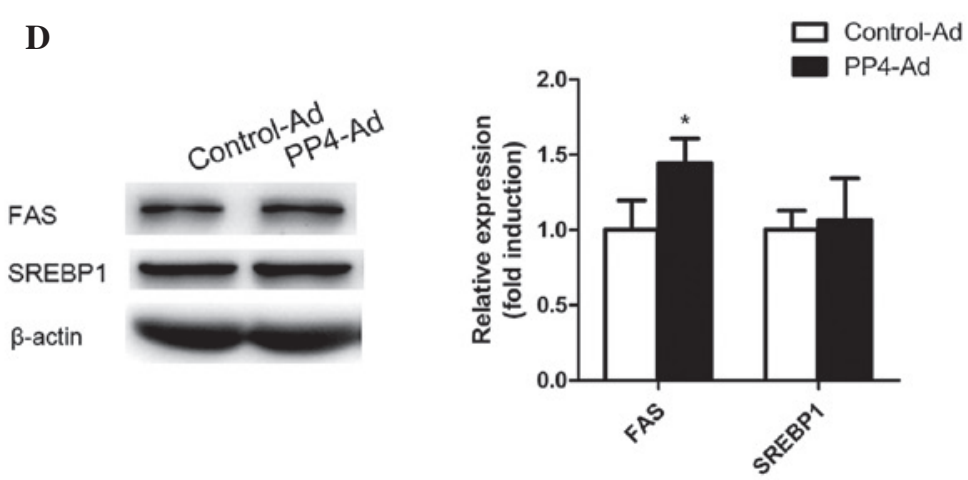

Figure 2. PP4 overexpression induces triglyceride accumulation in mouse primary hepatocytes through regulating the dephosphorylation of ACC1. (A) Mouse primary hepatocytes were transfected with adenovirus vectors containing only GFP or GFP-PP4 for $24 \mathrm{~h}$. (B) The GFP label was detected and PP4 levels were analyzed using western blot analysis. (C) Intracellular triglyceride content was measured using a triglyceride enzymatic assay kit. (D) The levels of FAS and SREBP1 were quantified using western blot analysis and densitometry analysis. Data are presented as the mean \pm standard deviation of three independent experiments. ${ }^{*} \mathrm{P}<0.05 ;{ }^{* *} \mathrm{P}<0.01 ;{ }^{* * *} \mathrm{P}<0.001$ vs. control $(\mathrm{n}=3)$. PP4, protein phosphatase 4; GFP, green fluorescent protein; Ad, adenovirus; FAS, fatty acid synthase; SREBP1, sterol regulatory element-binding protein.

also detected in the livers of db/db mice (Fig. 3B), suggesting that PP4 may be involved in triglyceride metabolism through regulating the dephosphorylation of ACC1.
PP4 dephosphorylates ACC1 directly through interacting with ACC1. The present study investigated whether PP4 was able to dephosphorylate ACC1 directly. 
A
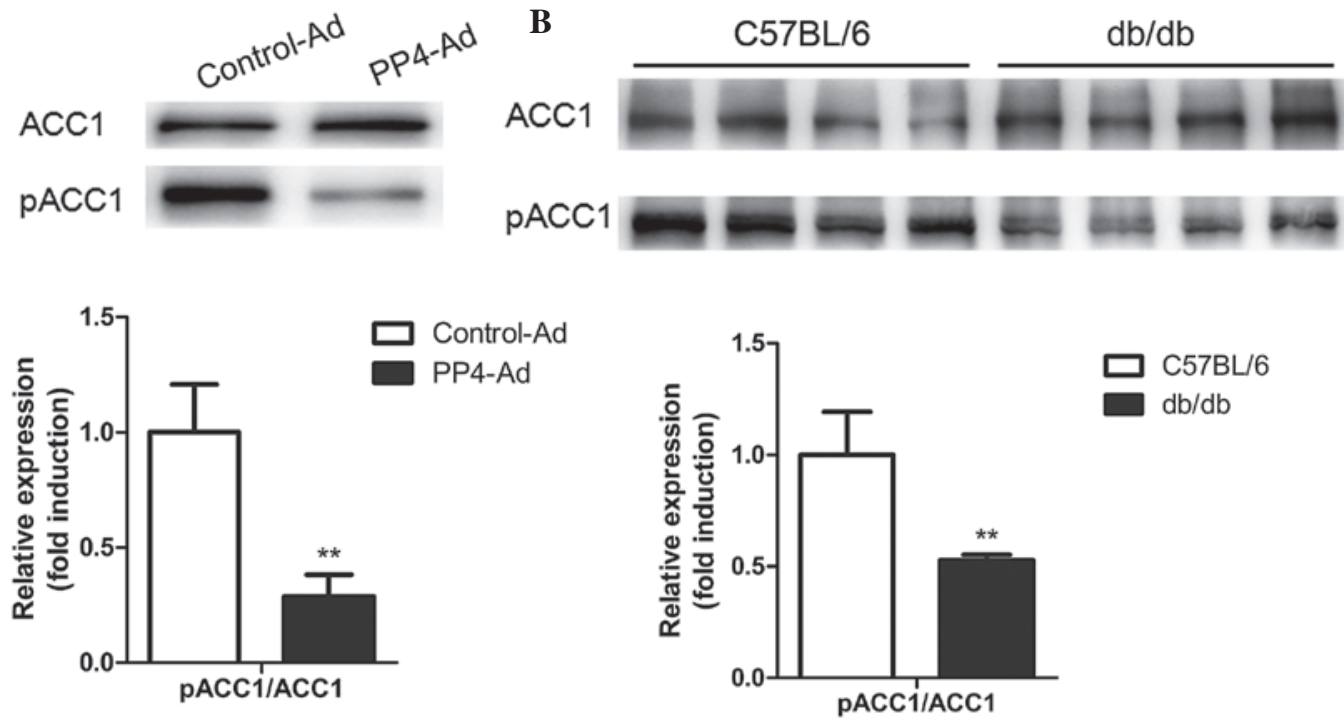

Figure 3. pACC1 levels decrease in the livers of db/db mice and mouse primary hepatocytes in response to PP4-Ad treatment. (A and B) ACC1 and pACC1-Ser79 were analyzed using western blot analysis in the mouse primary hepatocytes and the livers of $\mathrm{db} / \mathrm{db}$ mice. The ratio of pACC1-Ser79 to ACC1 was significantly decreased. " $\mathrm{P}<0.01$ vs. control $(\mathrm{n}=4)$. PP4, protein phosphatase 4; p, phosphorylated, ACC1, acetyl-CoA carboxylase 1; Ad, adenovirus.

A

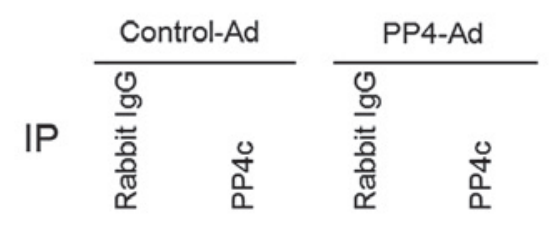

IB: pACC1

IB: PP4

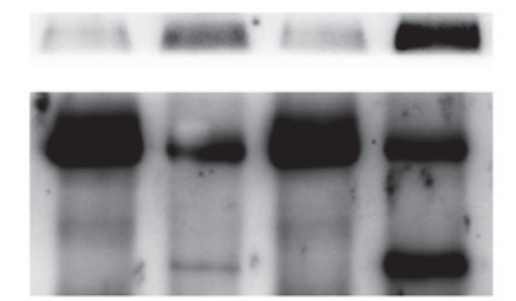

Control-Ad PP4-Ad
B

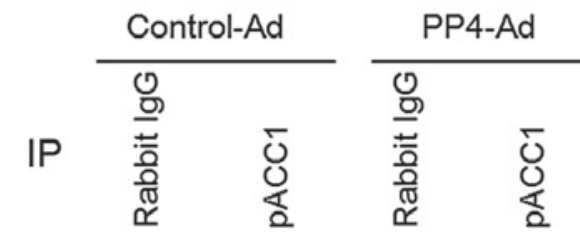

PACC1 IB: pACC1

$\lg G$

PP4

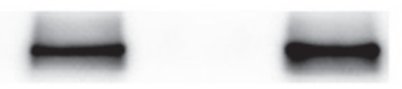

pACC1

$\lg G$

IB: PP4

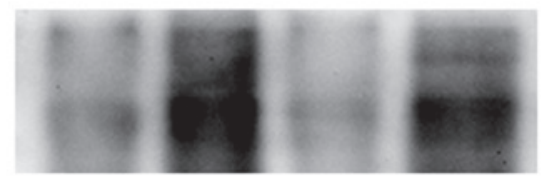

Figure 4. PP4 dephosphorylates ACC1 directly through interacting with ACC1. (A and B) HepG2 cells were transfected with control-Ad or PP4-Ad for 24 h. The direct interaction between PP4 and pACC1-Ser79 was confirmed using co-immunoprecipitation. PP4, protein phosphatase 4; p, phosphorylated; ACC1, acetyl-CoA carboxylase 1; Ad, adenovirus; IgG, immunoglobulin G.

Co-immunoprecipitation revealed that PP4 was capable of dephosphorylating ACC1 directly through direct interaction with ACC1 (Fig. 4A and B).

\section{Discussion}

ACC1 is a key enzyme involved in fatty acid biosynthesis from acetyl CoA to malonyl-CoA, which is an important step in fatty acid synthesis (21-23). ACC1 is highly expressed in tissues involved in lipogenesis, including the liver and adipose tissues (24). The activity of ACC1 is regulated by $\mathrm{Mn}^{2+}$, insulin, citrate and certain other conditions $(21,25,26)$. Previous studies of ACC1 have shown that $\mathrm{ACCl}$ is phosphorylated on approximately eight serine residues by six different protein kinases in hepatocytes $(9,27)$. However, the phosphorylation of ACC1 at Ser-79 by AMPK has been identified to be the main site and is capable of reducing the activity of ACC1 in vitro and in vivo $(27,28)$.
By contrast, dephosphorylation of ACC1 at Ser-79 has been found to increase the catalytic activity of ACC1. Evidence has shown that phosphorylated ACC1 is dephosphorylated by PP2Ac (8). Moreover, in the present study, PP4, a member of the PP2A family which only shares $65 \%$ amino acid homology with PP2A, was observed to directly dephosphorylate ACC1 at Ser-79 through interacting with $\mathrm{ACC} 1$, which increased the activity of ACC1, resulting in the accumulation of intracellular triglycerides in hepatocytes. However, when the hepatocytes were treated with a higher dose of PP4-Ad, the concentration of intracellular triglycerides markedly decreased (data not shown), suggesting that PP4 may be involved in other mechanisms in lipid metabolism.

As a ubiquitously expressed serine/threonine protein phosphatase, PP4 dephosphorylates a wide range of substrates at serine/threonine residues and is involved in various signaling pathways $(12-14,29)$. The findings of the present study widen the range of the biological effects of PP4. In the present study, PP4 
was observed to directly dephosphorylate the key active site of $\mathrm{ACC} 1$, resulting in an increase in FAS, a target of ACC1, as well as the biosynthesis and accumulation of intracellular triglycerides. However, no such effect was observed on SREBP1.

As a classic animal model, $\mathrm{db} / \mathrm{db}$ mice are widely used in studies of NAFLD and insulin resistance. In the present study, PP4 was observed to be highly expressed (2.5-fold) in the livers of $\mathrm{db} / \mathrm{db}$ mice and the ratio of $\mathrm{pACC} 1$ and $\mathrm{ACC} 1$ was found to be decreased, suggesting that PP4 may dephosphorylate $\mathrm{ACC} 1$ and increase $\mathrm{ACC} 1$ activity. This may suggest that PP4 is responsible, at least in part, for triglyceride accumulation in the livers of $\mathrm{db} / \mathrm{db}$ mice. However, $\mathrm{pACC} 1$ was also highly expressed in the livers of $\mathrm{db} / \mathrm{db}$ mice, indicating other factors may be involved in reducing the activity of $\mathrm{ACC} 1$ in lipid metabolism disorders.

A previous study showed that the majority of lipids (>90\%) are synthesized through de novo lipogenesis in cancer cells (30), suggesting that the biosynthesis of fatty acids is required for carcinogenesis. In the present study, ACC1, an important enzyme for de novo lipogenesis, was found to be strongly upregulated by PP4. Thus, PP4 may be a target for cancer therapy in the future.

In conclusion, the present study investigated the direct interaction between PP4 and pACC1-Ser79. PP4 was found to enhance ACC1 activity through dephosphorylation, leading to intracellular triglyceride accumulation. The results of the present study may provide insight into novel regulatory mechanisms of the activity of ACC1 in physiological and/or pathological lipogenesis. Moreover, the findings of the present study have enhanced the understanding of the functionality of PP4 in lipid metabolism. However, the versatile role of PP4 in cellular processes, as well as its physiological functions and underlying mechanisms, have yet to be elucidated.

\section{Acknowledgements}

The present study was supported by grants from the National Basic Research Program of China (no. 2012CB517502) and the National Natural Science Foundation of China (nos. 81270495, 81170381 and 81270887).

\section{References}

1. Collantes RS, Ong JP and Younossi ZM: The metabolic syndrome and nonalcoholic fatty liver disease. Panminerva Med 48: 41-48, 2006.

2. Ruhl CE and Everhart JE: Epidemiology of nonalcoholic fatty liver. Clin Liver Dis 8: 501-519, 2004.

3. Abu-Elheiga L, Matzuk MM, Kordari P, et al: Mutant mice lacking acetyl-CoA carboxylase 1 are embryonically lethal. Proc Natl Acad Sci USA 102: 12011-12016, 2005.

4. Chang HC, Seidman I, Teebor G and Lane MD: Liver acetyl CoA carboxylase and fatty acid synthetase: relative activities in the normal state and in hereditary obesity. Biochem Biophys Res Commun 28: 682-686, 1967.

5. Thampy KG and Wakil SJ: Regulation of acetyl-coenzyme A carboxylase. I. Purification and properties of two forms of acetyl-coenzyme A carboxylase from rat liver. J Biol Chem 263: 6447-6453, 1988.

6. Mabrouk GM, Helmy IM, Thampy KG and Wakil SJ: Acute hormonal control of acetyl-CoA carboxylase. The roles of insulin, glucagon, and epinephrine. J Biol Chem 265: 6330-6338, 1990.
7. Mohamed AH, Huang WY, Huang W, Venkatachalam KV and Wakil SJ: Isolation and characterization of a novel acetyl-CoA carboxylase kinase from rat liver. J Biol Chem 269: 6859-6865, 1994

8. Gaussin V, Hue L, Stalmans W and Bollen M: Activation of hepatic acetyl-CoA carboxylase by glutamate and $\mathrm{Mg}^{2+}$ is mediated by protein phosphatase-2A. Biochem J 316: 217-224, 1996.

9. Davies SP, Sim AT and Hardie DG: Location and function of three sites phosphorylated on rat acetyl-CoA carboxylase by the AMP-activated protein kinase. Eur J Biochem 187: 183-190, 1990.

10. Carling D and Hardie DG: The substrate and sequence specificity of the AMP-activated protein kinase. Phosphorylation of glycogen synthase and phosphorylase kinase. Biochim Biophys Acta 1012: 81-86, 1989.

11. Cohen PT, Philp A and Vázquez-Matin C: Protein phosphatase 4 - from obscurity to vital fuctions. FEBS Lett 579: 3278-3286, 2005.

12. Mourtada-Maarabouni M and Williams GT: Protein phosphatase 4 regulates apoptosis in leukemic and primary human T-cells. Leuk Res 1539-1551, 2009.

13. Jia S, Dai F, Wu D, et al: Protein phosphatase 4 cooperates with Smads to promote BMP signaling in dorsoventral patterning of zebrafish embryos. Dev Cell 22: 1065-1078, 2012.

14. Zhang X, Ozawa Y and Lee H: Histone deacetylase 3 (HDAC3) activity is regulated by interaction with protein serine/threonine phosphatase 4. Genes Dev 19: 827-839, 2005.

15. Hu MC, Tang-Oxley Q, Qiu WR, et al: Protein phosphatase $X$ interacts with c-Rel and stimulates c-Rel/nuclear factor kappaB activity. J Biol Chem 273: 33561-33565, 1998.

16. Wang R, Kong X, Cui A, et al: Sterol-regulatory-element-binding protein 1c mediates the effect of insulin on the expression of Cidea in mouse hepatocytes. Biochem J 430: 245-254, 2010.

17. Zhou Y, Jia S, Wang C, et al: FAM3A is a target gene of peroxisome proliferator-activated receptor gamma. Biochim Biophys Acta 1830: 4160-4170, 2013.

18. Han CC, Wang JW, Pan ZX, et al: Effect of liver X receptor activation on the very low density lipoprotein secretion and messenger ribonucleic acid level of related genes in goose primary hepatocytes. Poult Sci 90: 402-409, 2011.

19. Tang W, Ma Y, Jia L, Ioannou YA, Davies JP and Yu L: Genetic inactivation of NPC1L1 protects against sitosterolemia in mice lacking ABCG5/ABCG8. J Lipid Res 50: 293-300, 2009.

20. Naranmandura $\mathrm{H}, \mathrm{Xu} \mathrm{S}$, Koike $\mathrm{S}$, et al: The endoplasmic reticulum is a target organelle for trivalent dimethylarsinic acid (DMAIII)-induced cytotoxicity. Toxicol Appl Pharmacol 260: 241-249, 2012.

21. Hardie DG: Regulation of fatty acid synthesis via phosphorylation of acetyl-CoA carboxylase. Prog Lipid Res 28: 117-146, 1989.

22. Kim KH: Regulation of mammalian acetyl-coenzyme A carboxylase. Annu Rev Nutr 17: 77-99, 1997.

23. Shui JW, Hu MC and Tan TH: Conditional knockout mice reveal an essential role of protein phosphatase 4 in thymocyte development and pre-T-cell receptor signaling. Mol Cell Biol 27: 79-91, 2007.

24. Bianchi A, Evans JL, Iverson AJ, Nordlund AC, Watts TD and Witters LA: Identification of an isozymic form of acetyl-CoA carboxylase. J Biol Chem 265: 1502-1509, 1990.

25. Thampy KG and Wakil SJ: Activation of acetyl-CoA carboxylase. Purification and properties of a $\mathrm{Mn}^{2+}$-dependent phosphatase. J Biol Chem 260: 6318-6323, 1985.

26. Haystead TA and Hardie DG: Evidence that activation of acetyl-CoA carboxylase by insulin in adipocytes is mediated by a low-Mr effector and not by increased phosphorylation. Biochem J 240: 99-106, 1986

27. Hardie DG, Carling D and Sim ATR: The AMP-activated protein kinase: a multisubstrate regulator of lipid metabolism. Trends Biochem Sci 14: 20-23, 1989.

28. Ha J, Daniel S, Broyles SS and Kim KH: Critical phosphorylation sites for acetyl-CoA carboxylase activity. J Biol Chem 269: 22162-22168, 1994.

29. Yoon YS, Lee MW, Ryu D, et al: Suppressor of MEK null (SMEK)/protein phosphatase 4 catalytic subunit (PP4C) is a key regulator of hepatic gluconeogenesis. Proc Natl Acad Sci USA 107: 17704-17709, 2010.

30. Milgraum LZ, Witters LA, Pasternack GR and Kuhajda FP: Enzymes of the fatty acid synthesis pathway are highly expressed in in situ breast carcinoma. Clin Cancer Res 3: 2115-2120, 1997. 